\title{
Mathematical Modeling Analysis to Simulate the Dynamics of Immune Cells, HIV, and Tuberculosis
}

\author{
Rumana Abmed *a Mabbubur Rabman ${ }^{b}$ \\ aDepartment of Biology, the City College of the City University of New York, New York, NY \\ bDepartment of Mathematics and Statistics, University of North Florida, Jacksonville, FL
}

Student: rumed1992@gmail.com*

Mentor:mrahman@,unf.edu

\begin{abstract}
The dynamics of immune cells, HIV, and tuberculosis can be described by a system of differential equations. We developed the formulations for this dynamical system. To evaluate the system as time goes to infinity, we investigated the equilibrium solutions. We established the criteria for stability based on the characteristics of the Jacobian matrix associated with the dynamical system. To further investigate the stability of the system, we developed phase plane diagrams for the sets of assumed values of the parameters. We have investigated the curves for different values of the starting conditions of immune cells and the antigens. Along the curves, we observed the growth and decay processes. The stability of the system has been established by examining the phase plane diagrams as the solution approaches the equilibrium point. Based on phase diagrams, both stable and unstable systems have been simulated and examined in this study. Finally, we developed and evaluated the graphs for the unsteady variations of immune cells, HIV, and tuberculosis to see how the antigens grow because of the diminishing effects of immune cells in the system as time increases.
\end{abstract}

\section{KEYWORDS}

Mathematical Biology; Infectious Disease Modeling; Dynamical System; Simulation of Immune Cells and Antigens

\section{INTRODUCTION}

We performed mathematical analyses to investigate the growth and decay of the population of blood cells and antigens in a host. These analyses lead to the establishment of mathematical models that can be used to simulate the antigenic variations and impact of infectious disease on a human population. ${ }^{1}$ An in-depth analysis includes investigation of the formulations for the population that are infected and susceptible. ${ }^{2} \mathrm{~A}$ major concern is the growth of infection caused by antigens on an HIV patient. There are declining trends on the mortality and morbidity rates of tuberculosis, ${ }^{3}$ however, an HIV patient is highly susceptible to the infection caused by other agents such as tuberculosis.

We developed formulations to evaluate the effects of an infection on an HIV patient. An HIV patient is infected with a virus called lentivirus, which is a subgroup of retrovirus. The infection caused by an opportunistic virus or a bacteria can complicate the conditions of an HIV patient. ${ }^{4}$ A co-infection of $13 \%$ occurred with HIV patients in 2011.5 There will be stress on the immune cells already engaged in fighting the HIV virus. The immune response will occur from the body due to the presence of a new opportunistic agent. An immune system of a healthy and HIV-free person is able to overcome the effects of any pathogen by eliminating it through the immune reactions. However, the pre-occupied and stressed immune system will have to deal with the new pathogen in an HIV patient.

The system in an HIV patient with co-infection will involve the dynamics of the immune cells, the HIV virus, and a new opportunistic pathogen. HIV patients are vulnerable to the infections caused by certain opportunistic pathogens. These pathogens take the opportunity provided by a weak immune system. One such opportunistic pathogen is a bacterium called tuberculosis. An earlier study was performed to develop formulations for the investigation of the long-term dynamics of tuberculosis. ${ }^{3}$ This study focuses on the formulation and investigation of the dynamical system in an HIV patient infected by tuberculosis. In addition, the dynamical system will involve the processes of interaction within the host.

The infections caused by HIV lead to the deaths of infected cells due to the pathological activity (cytopathicity) of HIV or by immune reactions. ${ }^{6} \mathrm{HIV}$ is a group of RNA viruses (ribonucleic acid) that insert a DNA (deoxyribonucleic acid) copy of their genome into the host cell in order to replicate. These processes can lead to a complicated dynamic involving the growth and decay of HIV. Further complications occur with the introduction of a new pathogen. In this study, we developed mathematical relationships to represent the effects of HIV and the effects of tuberculosis on the immune response. The net specific growth rates will include the terms that will represent the interaction of immune cells, HIV virus, and tuberculosis. 
Mathematical modeling analyses have been widely used for the simulation of HIV infection. The population model formulation for the transmission of HIV included susceptible and infected individuals in a community, We performed a study to review the technical approaches for the development of the mathematical models on the population dynamics associated with HIV infection. ${ }^{8}$ The epidemiological models were developed to simulate the transmission dynamics of the spread of HIV. The investigation of HIV in a host requires development of governing equations involving rate constants as parameters for growth, decay, and mass action. ${ }^{9}$ The dynamical system for HIV included the development of the formulations for the concentrations of infected T-cells, uninfected T-cells, and viruses. ${ }^{10}$ The formulations for within-host models included viruses as well as susceptible and infected target cells. In the diversity threshold model, the formulations were developed for the density of virus strains and non-specific T-cells. The dynamics of mature and immature T-cells were formulated in a host with HIV infection. ${ }^{11}$ We performed a mathematical modeling analysis for HIV pathogenesis and treatment. ${ }^{12}$ Through this modeling study, HIV dynamics, disease progression, and therapy were investigated.

In the aforementioned studies, we modeled T-cells and HIV for the investigation of the system in a host and for the investigation of the transmission in the population of a community. The present modeling analysis entails the investigation of the evolution of non-specific T-cells, HIV, and tuberculosis in an infected host. The objectives of the study are as follows.

- Develop the dynamic formulation for non-specific T-cells, HIV, and tuberculosis.

- Derive the equilibrium solution.

- Perform a stability analysis based on the equilibrium solution.

- Perform analysis of the phase plane diagrams for the dynamical system.

- Simulate an example problem.

\section{MODEL FORMULATION}

When attacked by the HIV virus, T-cells are able to fight off the invasion for a period of time. Simultaneously, the HIV virus destroys infection-fighting T-cells. As a result, the immune system becomes less effective at fighting disease (including the spread of HIV). The present model formulation incorporates the forces involved in this process. The forces of growth and decay are included in the formulation, along with the effects on the system involving non-specific T-cells, HIV, and tuberculosis.

T-cells, or immune cells, are an essential and natural component of the human immune system. The primary job of T-cells is to attack and destroy cancerous cells, cells infected with a virus, and in general, any cell containing foreign antigens. The typical Tcell count in a healthy person is about 1000-2000 per cubic millimeter. T-cells are created in the lymphatic tissues. These tissues normally produce T-cells at a uniform (constant) rate, but when a need is detected to combat a virus, additional T-cells are created. T-cells only live for a finite period of time. HIV is measured as a viral load in a sample of blood. The viral load is the number of HIV virus particles in a milliliter of blood. The bacterium for tuberculosis is measured in Colony Forming Units $(\mathrm{CFU})$. The $\mathrm{CFU}$ is defined to be the number of bacteria in a sample (milliliter) of blood. ${ }^{13}$

Let $T$ be the quantity of immune cells (non-specific T-cells), $V_{H}$ be the quantity of HIV, and $V_{T}$ be the quantity of tuberculosis. In the absence of immune cells, the population sizes $V_{H}$ and $V_{T}$ will grow exponentially. ${ }^{6}$ Let's formulate the differential equation for the three populations quantified by the three dependent variables: $T, V_{H}$, and $V_{T}$. The population growth equations for $T, V_{H}$, and $V_{T}$ with the corresponding net specific growth rates can be written as

$$
\begin{gathered}
\frac{1}{T} \frac{d T}{d t}=\mu_{1}, \\
\frac{1}{V_{H}} \frac{d V_{H}}{d t}=\mu_{2}, \\
\frac{1}{V_{T}} \frac{d V_{T}}{d t}=\mu_{3}
\end{gathered}
$$

Equation 1.

Equation 2.

Equation 3.

Here $\mu_{1}, \mu_{2}$, and $\mu_{3}$ are net specific growth rates for non-specific T-cells, HIV, and tuberculosis, respectively. The net specific growth rates are dependent on the forces of growth and decay as well as their effects, assuming that the rates of change of the populations are proportional ${ }^{14}$ It is assumed that the net specific growth rate $\mu_{l}$ will be proportional to the population of $V_{H}$ and $V_{T}$, as will the individual net growth rate. The number of T-cells does not increase in an unbounded fashion. The individual net 
growth rate for the immune cells is proportional is to a logistic term: $\alpha\left(1-\frac{T}{T_{\max }}\right)$. Here $T_{\max }$ is the least upper bound for the population of T-cells. HIV and tuberculosis will have adverse effects on the immune cells. Based on the aforementioned assumptions, we can write the net specific growth rate, $\mu_{1}$, for $T$ as

where

$$
\mu_{1}=\alpha\left(1-\frac{T}{T_{\max }}\right)-\beta V_{H}-\gamma V_{T}
$$

$\alpha=$ net growth rate of $T$,

$\beta=$ rate of the destructive effects on $T$ of the interaction of $T$ and $V_{H}$, and

$\gamma=$ rate of effects of the interaction of $T$ and $V_{T}$ on $T$.

Substituting Equation 4 for $\mu_{1}$ in Equation 1, we can write

$$
\frac{d T}{d t}=\alpha T\left(1-\frac{T}{T_{\max }}\right)-\beta T V_{H}-\gamma T V_{T}
$$

Equation 5.

Tuberculosis is the most common co-infection in patients with HIV We found that the HIV load was significantly higher in patients co-infected with tuberculosis, ${ }^{15}$ so it can be assumed that tuberculosis will cause an increase in the growth of HIV. It is known that the immune cells will cause a decrease in HIV. Therefore, the net specific growth rate for HIV can be written as

where

$$
\mu_{2}=-\xi T+\eta+\zeta V_{T}
$$

Equation 6.

$\xi=$ the rate of the effects of the interaction of $T$ and $V_{H}$ on $V_{H}$,

$\eta=$ net growth rate of $V_{H}$, and

$\zeta=$ rate of the effects of the interaction of $V_{H}$ and $V_{T}$ on $V_{H}$.

Substituting Equation 6 for $\mu_{2}$ in Equation 2, we can write the governing differential equations for $V_{H}$ as follows.

$$
\frac{d V_{H}}{d t}=-\xi T V_{H}+\eta V_{H}+\zeta V_{H} V_{T}
$$

Equation 7.

We investigated the impact of HIV on tuberculosis. The study showed that tuberculosis increases in patients co-infected with HIV. ${ }^{16}$ As usual, immune cells have negative effects on the population of tuberculosis. The net specific growth rate for tuberculosis can be written as

$$
\mu_{3}=-\varepsilon T+\delta V_{H}+\sigma
$$

Equation 8.

where

$\varepsilon=$ the rate of the effects of the interaction of $T$ and $V_{T}$ on $V_{T}$,

$\delta=$ the rate of the effects of the interaction of $V_{H}$ and $V_{T}$ on $V_{T}$, and

$\sigma=$ net growth rate of $V_{T}$.

Substituting Equation 8 for $\mu_{3}$ in Equation 3, we can write the governing differential equation for $V_{T}$ as

$$
\frac{d T_{T}}{d t}=-\varepsilon T V_{T}+\delta V_{H} V_{T}+\sigma V_{T}
$$

Equation 9.

The system of governing differential equations representing the dynamical system for the growth/decay of the population of immune cells, HIV, and tuberculosis can be written as follows.

$$
\begin{gathered}
\frac{d T}{d t}=\alpha T\left(1-\frac{T}{T_{\max }}\right)-\beta T V_{H}-\gamma T V_{T} \\
\frac{d T_{H}}{d t}=-\xi T V_{H}+\eta V_{H}+\zeta V_{H} V_{T} \\
\frac{d V}{d t}=-\varepsilon T V_{T}+\delta V_{H} V_{T}+\sigma V_{T}
\end{gathered}
$$

Equation 11.

Equation 12. 
The model remains appropriate as long as each population does not become negative. The parameters $\alpha, \eta$, and $\sigma$ define the growth rates for $T, V_{H}$, and $V_{T}$, respectively. Starting with $T(0)>0$ the only way $T$ can be negative is if $\frac{1}{T} \frac{d T}{d t}<0$ at some time $t$ for which $T(t)=0$. But if $T=0$ the first ordinary differential equation defined by Equation 10 yields $\frac{1}{T} \frac{d T}{d t}=\alpha>0$. This guarantees that $T(t)>0$ for all $t$. Similarly, it can be proven that $V_{H}>0$ and $V_{T}>0$ for all $t$.

As described earlier, a number of parameters $(\alpha, \beta, \gamma, \xi, \eta, \zeta, \varepsilon, \delta$, and $\sigma)$ are used in defining the specific growth rates and interaction of T-cells, HIV, and tuberculosis. The solution will depend on the values of these parameters. As shown in the next section, the equilibrium solution and the stability of the system depend on the parameters describing the growth, decay, and interaction among the T-cells, HIV, and tuberculosis. These parameters are rate constants and are expressed as units over time. The unsteady solutions of the dynamical system (Equations 10, 11, and 12), solutions for equilibrium condition, and stability analysis results have been computed using Maple, based on the derivations. ${ }^{17}$

\section{EQUILIBRIUM SOLUTION}

The equilibrium solution is obtained at a steady state condition that is found as $t \rightarrow \infty$. If we set each derivative equal to zero, we obtain three equations with the equilibrium condition:

$$
\begin{gathered}
\alpha T\left(1-\frac{T}{T_{\max }}\right)-\beta T V_{H}-\gamma T V_{T}=0 \\
-\xi T V_{H}+\eta V_{H}+\zeta V_{H} V_{T}=0, \\
-\varepsilon T V_{T}+\delta V_{H} V_{T}+\sigma V_{T}=0 .
\end{gathered}
$$

After examining the above equations, we find one of the two equilibrium conditions, $\left(T^{o}, V_{H}^{o}, V_{T}^{o}\right)=(0,0,0)$.

The equilibrium Equations 13, 14, and 15 can be simplified and written in the form

$$
\begin{gathered}
\alpha\left(1-\frac{T}{T_{\max }}\right)-\beta V_{H}-\gamma V_{T}=0 \\
-\xi T+\eta+\zeta V_{T}=0, \\
-\varepsilon T+\delta V_{H}+\sigma=0 .
\end{gathered}
$$

After solving the above three equations for three population sizes, we get the solution of the equilibrium equations:

$$
\begin{gathered}
T^{*}=\frac{T_{\max }(\alpha \delta \zeta+\beta \sigma \zeta+\delta \eta \gamma)}{T_{\max }(\beta \varepsilon \zeta+\delta \gamma \xi)+\alpha \delta \zeta,} \\
V_{H}^{*}=\frac{T_{\max }(\alpha \varepsilon \zeta+\varepsilon \eta \gamma-\gamma \sigma \xi)-\alpha \sigma \zeta}{T_{\max }(\beta \varepsilon \zeta+\delta \gamma \xi)+\alpha \delta \zeta}, \\
V_{T}^{*}=\frac{T_{\max }(\alpha \delta \xi-\beta \varepsilon \eta+\beta \sigma \xi)-\alpha \delta \eta}{T_{\max }(\beta \varepsilon \zeta+\delta \gamma \xi)+\alpha \delta \zeta} .
\end{gathered}
$$

Equation 19.

Equation 20.

Equation 21.

The above solutions show the second equilibrium point $\left(T^{*}, V_{H}^{*}, V_{T}^{*}\right)$ for the dynamical system defined by the Equations 19 , 20 , and 21 .

\section{STABILITY ANALYSIS}

The differential equations representing the dynamical system for T-cells, HIV, and tuberculosis can be denoted

$$
\begin{aligned}
& \frac{d T}{d t}=f\left(T, V_{H}, V_{T}\right) \\
& \frac{d V_{H}}{d t}=g\left(T, V_{H}, V_{T}\right)
\end{aligned}
$$




$$
\frac{d V_{T}}{d t}=h\left(T, V_{H}, V_{T}\right)
$$

Equation 24.

where

$$
\begin{aligned}
& f\left(T, V_{H}, V_{T}\right)=\alpha T\left(1-\frac{T}{T_{\max }}\right)-\beta T V_{H}-\gamma T V_{T} \\
& g\left(T, V_{H}, V_{T}\right)=-\xi T V_{H}+\eta V_{H}+\zeta V_{H} V_{T} \\
& h\left(T, V_{H}, V_{T}\right)=-\varepsilon T V_{T}+\delta V_{H} V_{T}+\sigma V_{T}
\end{aligned}
$$

The stability of the system can be examined by evaluating the stability matrix, a Jacobian matrix, $J$, defined as

$$
J=\left[\begin{array}{ccc}
\frac{\partial f}{\partial T} & \frac{\partial f}{\partial V_{H}} & \frac{\partial f}{\partial V_{T}} \\
\frac{\partial g}{\partial T} & \frac{\partial g}{\partial V_{H}} & \frac{\partial g}{\partial V_{T}} \\
\frac{\partial h}{\partial T} & \frac{\partial h}{\partial V_{H}} & \frac{\partial h}{\partial V_{T}}
\end{array}\right] .
$$

By evaluating the derivatives, the Jacobian matrix, $J$, can be written as

$$
J\left(T, V_{H}, V_{T}\right)=\left[\begin{array}{ccc}
\alpha\left(1-\frac{T}{T_{\max }}\right)-\alpha \frac{T}{T_{\max }}-\beta V_{H}-\gamma V_{T} & -\beta T & -\gamma T \\
-\xi V_{H} & \eta-\xi T+\zeta V_{T} & \zeta V_{H} \\
-\varepsilon V_{T} & \delta V_{T} & \sigma-\varepsilon T+\delta V_{H}
\end{array}\right] .
$$

\section{STABILITY AT THE FIRST EQUILIBRIUM POINT}

Substituting the first equilibrium point $\left(T^{o}, V_{H}^{o}, V_{T}^{o}\right)=(0,0,0)$ into the Jacobian matrix, we get

$$
J(0,0,0)=\left[\begin{array}{lll}
\alpha & 0 & 0 \\
0 & \eta & 0 \\
0 & 0 & \sigma
\end{array}\right]
$$

The above matrix is diagonal. The determinant and trace are $\operatorname{det}(J(0,0,0))=\alpha \eta \sigma$ and $\operatorname{tr}(J(0,0,0))=\alpha+\eta+\sigma$, respectively.

To investigate the stability, check the trace and determinant. For the system at the first equilibrium to be stable, the determinant must be greater than 0 and the trace must be less than 0 . These conditions are written as $\alpha \eta \sigma>0$ and $\alpha+\eta+\sigma<0$.

Since the Jacobian matrix at the first equilibrium point is diagonal, its eigenvalues, $\lambda_{1}, \lambda_{2}, \lambda_{3}$, are the diagonal elements. Therefore $\lambda_{1}=\alpha, \lambda_{2}=\eta$, and $\lambda_{3}=\sigma$.

For the system to be stable, the eigenvalues must be negative. With the parameters $\alpha$, $\eta$, and $\sigma$ having positive values, the determinant, trace, and the eigenvalues are all greater than 0 . This indicates that the system is not stable at the first equilibrium point. 


\section{STABILITY AT THE SECOND EQUILIBRIUM POINT}

Substituting the second equilibrium point $\left(T^{*}, V_{H}^{*}, V_{T}^{*}\right)$ into the Jacobian matrix, we get

$$
J\left(T^{*}, V_{H}^{*}, V_{T}^{*}\right)=\left[\begin{array}{ccc}
\alpha\left(1-\frac{\hat{\alpha}}{p}\right)-\frac{1}{p}(\alpha \hat{\alpha}+\beta \hat{\beta}+\gamma \hat{\gamma}) & -\beta \frac{T_{m} \hat{\alpha}}{p} & -\gamma \frac{T_{m} \hat{\alpha}}{p} \\
-\xi \frac{\hat{\beta}}{p} & -\xi \frac{T_{m} \hat{\alpha}}{p}+\zeta \frac{\hat{\gamma}}{p}+\eta & \zeta \frac{\hat{\beta}}{p} \\
-\varepsilon \frac{\hat{\gamma}}{p} & \delta \frac{\hat{\gamma}}{p} & -\varepsilon \frac{T_{m} \hat{\alpha}}{p}+\delta \frac{\hat{\beta}}{p}+\sigma
\end{array}\right],
$$

where

$\hat{\alpha}=\alpha \delta \zeta+\beta \sigma \zeta+\delta \eta \gamma$

$\hat{\beta}=T_{m}(\alpha \varepsilon \zeta+\varepsilon \eta \gamma-\gamma \sigma \xi)-\alpha \sigma \zeta$,

$\hat{\gamma}=T_{m}(\alpha \delta \xi-\beta \varepsilon \eta+\beta \sigma \xi)-\alpha \delta \eta$, and

$p=T_{m}(\beta \varepsilon \zeta+\delta \gamma \xi)+\alpha \delta \zeta$.

The determinant of the Jacobian at the second equilibrium point is $\operatorname{det}\left(J\left(T^{*}, V_{H}^{*}, V_{T}^{*}\right)\right)=\frac{\hat{\alpha} \hat{\beta} \hat{\gamma}}{p^{2}}$.

The second equilibrium point is stable if $\operatorname{det}\left(J\left(T^{*}, V_{H}^{*}, V_{T}^{*}\right)\right)>0$. This implies that $\frac{\hat{\alpha} \hat{\beta} \hat{\gamma}}{p^{2}}>0$.

The trace of the Jacobian matrix at the second equilibrium point is

$$
\operatorname{tr}\left(J\left(T^{*}, V_{H}^{*}, V_{T}^{*}\right)\right)=\alpha\left(1-\frac{\hat{\alpha}}{p}\right)-\frac{1}{p}\left(\alpha \hat{\alpha}+\beta \hat{\beta}+\gamma \hat{\gamma}+\xi T_{m} \hat{\alpha}-\zeta \hat{\gamma}+\varepsilon T_{m} \hat{\alpha}-\delta \hat{\beta}\right)+\eta+\sigma .
$$

For stability, the trace of the Jacobian matrix should be less than 0 . That is

$$
\alpha\left(1-\frac{\hat{\alpha}}{p}\right)-\frac{1}{p}\left(\alpha \hat{\alpha}+\beta \hat{\beta}+\gamma \hat{\gamma}+\xi T_{m} \hat{\alpha}-\zeta \hat{\gamma}+\varepsilon T_{m} \hat{\alpha}-\delta \hat{\beta}\right)+\eta+\sigma<0
$$

The eigenvalues, $\lambda_{1}, \lambda_{2}, \lambda_{3}$, can be obtained by setting $\operatorname{det}(J-\lambda I)=0$. The matrix $J-\lambda I$ can be written as

$$
J\left(T^{*}, V_{H}^{*}, V_{T}^{*}\right)-\lambda I=\left[\begin{array}{ccc}
\tilde{\alpha} & -\beta \frac{T_{m} \hat{\alpha}}{p} & -\gamma \frac{T_{m} \hat{\alpha}}{p} \\
-\xi \frac{\hat{\beta}}{p} & \tilde{\eta} & \xi \frac{\hat{\beta}}{p} \\
-\varepsilon \frac{\hat{\gamma}}{p} & \delta \frac{\hat{\gamma}}{p} & \tilde{\sigma}
\end{array}\right],
$$

where

$$
\begin{aligned}
& \tilde{\alpha}=\alpha\left(1-\frac{\hat{\alpha}}{p}\right)-\frac{1}{p}(\alpha \hat{\alpha}+\beta \hat{\beta}+\gamma \hat{\gamma})-\lambda I, \\
& \tilde{\eta}=-\xi \frac{T_{m} \hat{\alpha}}{p}+\xi \frac{\hat{\gamma}}{p}+\eta-\lambda I, \text { and }
\end{aligned}
$$




$$
\tilde{\sigma}=-\varepsilon \frac{T_{m} \hat{\alpha}}{p}+\delta \frac{\hat{\beta}}{p}+\sigma-\lambda I \text {. }
$$

The characteristic equation for eigenvalues can be obtained by writing $\operatorname{det}\left(J\left(T^{*}, V_{H}^{*}, V_{T}^{*}\right)-\lambda I\right)=0$. By evaluating the determinant, the characteristic equation for eigenvalues can be written in a cubic polynomial form as

$$
\lambda^{3}+u \lambda^{2}+v \lambda+w=0
$$

Equation 25.

where

$$
\begin{gathered}
u=\frac{b}{a}, \\
v=\frac{c}{a}, \\
w=\frac{d}{a}
\end{gathered}
$$$$
a=-\left[\frac{T_{\max }(\beta \varepsilon \zeta+\delta \gamma \xi)+\alpha \delta \zeta}{p}\right]^{2}
$$$$
b=\frac{-T_{m} \alpha^{2} \delta \zeta(\beta \varepsilon \zeta+\delta \gamma \xi)-\alpha(\beta \sigma \zeta+\delta \eta \gamma) p-\alpha^{3} \delta^{2} \zeta^{2}}{p^{2}},
$$$$
c=c_{1}+c_{2}+c_{3}+c_{4}+c_{5}+c_{6} \text {, }
$$$$
c_{1}=\frac{T_{\max }^{2} \alpha^{2} \delta \xi \zeta(\beta \varepsilon \zeta+\delta \varepsilon \gamma)+T_{\max }^{2} \alpha \varepsilon \xi \zeta^{2}\left(\alpha \delta^{2}+\beta^{2} \sigma\right)+T_{\max }^{2}(\gamma+\zeta)\left(\alpha \varepsilon \eta \gamma \xi \delta^{2}-\alpha \beta \delta \eta \zeta \varepsilon^{2}+\beta \delta \varepsilon \eta \gamma \sigma \xi\right)}{p^{2}},
$$$$
c_{2}=\frac{T_{\max }^{2} \beta \delta \varepsilon \eta \gamma \sigma \xi \zeta+T_{\max }^{2}(\eta+\sigma)\left(2 \alpha \beta \delta \varepsilon \gamma \xi \zeta+\varepsilon \gamma \sigma \xi \zeta \beta^{2}\right)}{p^{2}},
$$$$
c_{3}=\frac{T_{\max }(\varepsilon \zeta-\gamma \xi)\left(T_{m} \alpha \beta \delta \sigma \xi \zeta-T_{m} \beta \delta \varepsilon \gamma \eta^{2}+\alpha \beta \delta \eta \sigma \zeta\right)}{p^{2}},
$$

$c_{4}=\frac{-T_{\max }^{2}\left[\alpha \sigma \zeta\left(\alpha \delta^{2} \xi^{2}+\eta \beta^{2} \varepsilon^{2}\right)+\beta \gamma\left(\beta \zeta \sigma^{2} \xi^{2}+\delta \gamma \varepsilon^{2} \eta^{2}\right)\right]-T_{\max } \alpha \zeta(\beta \sigma \xi \zeta+\delta \varepsilon \eta \gamma)(\alpha \delta+\beta \sigma)}{p^{2}}$,

$c_{5}=\frac{-T_{\max }^{2} \beta \delta \gamma \sigma \xi^{2}(\eta \gamma+\sigma \zeta)-T_{\max } \alpha \delta\left[\alpha \delta \zeta^{2}(\varepsilon \eta+\sigma \xi)+\beta \xi \sigma^{2} \zeta^{2}+\delta \varepsilon \eta^{2} \gamma^{2}\right]}{p^{2}}$,

$c_{6}=\frac{-T_{\max } \alpha \eta \gamma \zeta \delta^{2}(\varepsilon \eta-\sigma \xi)+\eta \sigma \alpha^{2} \delta^{2} \zeta^{2}}{p^{2}}$,

$d=d_{1}+d_{2}+d_{3}+d_{4}+d_{5}+d_{6}$,

$d_{1}=\frac{T_{\max }^{2} \delta \xi \alpha^{2} \zeta^{2}(\alpha \delta \xi-\beta \varepsilon \eta)+\delta \zeta \alpha^{2}(\beta \sigma \zeta+\delta \eta \gamma)\left(2 T_{\max }^{2} \varepsilon \xi+\eta \sigma\right)}{p^{2}}$,

$d_{2}=\frac{T_{\max }^{2} \beta \delta \gamma \sigma \xi(2 \varepsilon \eta-\sigma \xi)(2 \alpha \zeta+\eta \gamma)}{p^{2}}$, 


$$
\begin{aligned}
& d_{3}=\frac{T_{\max }(\varepsilon \eta-\sigma \xi)\left[T_{m} \eta \gamma\left(\alpha \gamma \xi \delta^{2}-\varepsilon \sigma \zeta \beta^{2}\right)+\alpha\left(\beta^{2} \sigma^{2} \zeta^{2}-\delta^{2} \eta^{2} \gamma^{2}\right)\right]}{p^{2}}, \\
& d_{4}=\frac{T_{\max }^{2}\left[\varepsilon \eta \gamma \xi \zeta \beta^{2} \sigma^{2}-\alpha \sigma \zeta\left(\alpha \gamma \xi^{2} \delta^{2}+\eta \zeta \beta^{2} \varepsilon^{2}\right)\right]}{p^{2}}, \\
& d_{5}=\frac{T_{\max }^{2}\left[\alpha \beta \varepsilon \zeta\left(\beta \xi \zeta \sigma^{2}-2 \delta \varepsilon \gamma \eta^{2}\right)-\beta \gamma\left(\beta \zeta \sigma^{3} \xi^{2}-\delta \gamma \varepsilon^{2} \eta^{3}\right)\right]}{p^{2}}, \text { and } \\
& d_{6}=\frac{-T_{\max }\left[\alpha^{3} \delta^{2} \zeta^{2}(\varepsilon \eta+\sigma \xi)+2 \delta \zeta \alpha^{2}\left(\beta \xi \zeta \sigma^{2}+\delta \varepsilon \gamma \eta^{2}\right)\right]+\eta \sigma \alpha^{3} \delta^{2} \zeta^{2}}{p^{2}} .
\end{aligned}
$$

The function in the left side of the characteristic equation, Equation 25, for eigenvalues is a polynomial of degree three. This third degree polynomial has at most three roots representing the three eigenvalues of the Jacobian matrix. The equilibrium point $\left(T^{*}, V_{H}^{*}, V_{T}^{*}\right)$ will be stable if all the eigenvalues of the Jacobian matrix are negative (if real) or have negative real parts (if complex). So, for stable equilibrium, we need $\operatorname{Re} \lambda_{i}<0, \forall i$.

The general criteria, called Routh-Hurwitz criteria, ${ }^{18}$ (described in Appendix A) can be used to determine whether all the roots of the characteristic equation (a third degree polynomial equation) for eigenvalues have negative real part or not. For the above cubic polynomial equation, evaluated at the eigenvalues, Routh-Hurwitz criteria are $u>0, v>0, w>0$, and $u v>w$.

\section{ANALYSIS OF PHASE DIAGRAMS}

The mathematical study of dynamical systems entails investigation of the changes of the topology of the phase plane. Assigning the assumed values of the parameters, we obtained and analyzed the phase diagrams to observe the stability of the solution. The values of the parameters were assigned in order to investigate the topology of the phase plane diagrams. Based on the assumed values of the parameters, the phase plane diagrams were developed using XPPAU'T software. ${ }^{19}$

Through the investigation of the phase plane diagrams, we evaluated the stability of the present dynamical system. Figures 1 through $\mathbf{6}$ show the phase diagrams for different values of the parameters. The phase diagrams showing a set of closed curves are illustrated in Figures 1 and 2. These curves represent the variations of T-cells with HIV (Figure 1) and the variations of T-cells with tuberculosis (Figure 2) for different initial conditions. Each curve corresponds to an initial condition, which was defined graphically through XPPAUT. The phase plane diagrams with curves around a center represent a neutrally stable system, ${ }^{20}$ so Figures 1 and 2 are examples of phase plane diagrams for neutrally stable systems showing a cycle of growth and decay associated with T-cells, HIV, and tuberculosis. Here, the system approaches the equilibrium point at the center with stability.

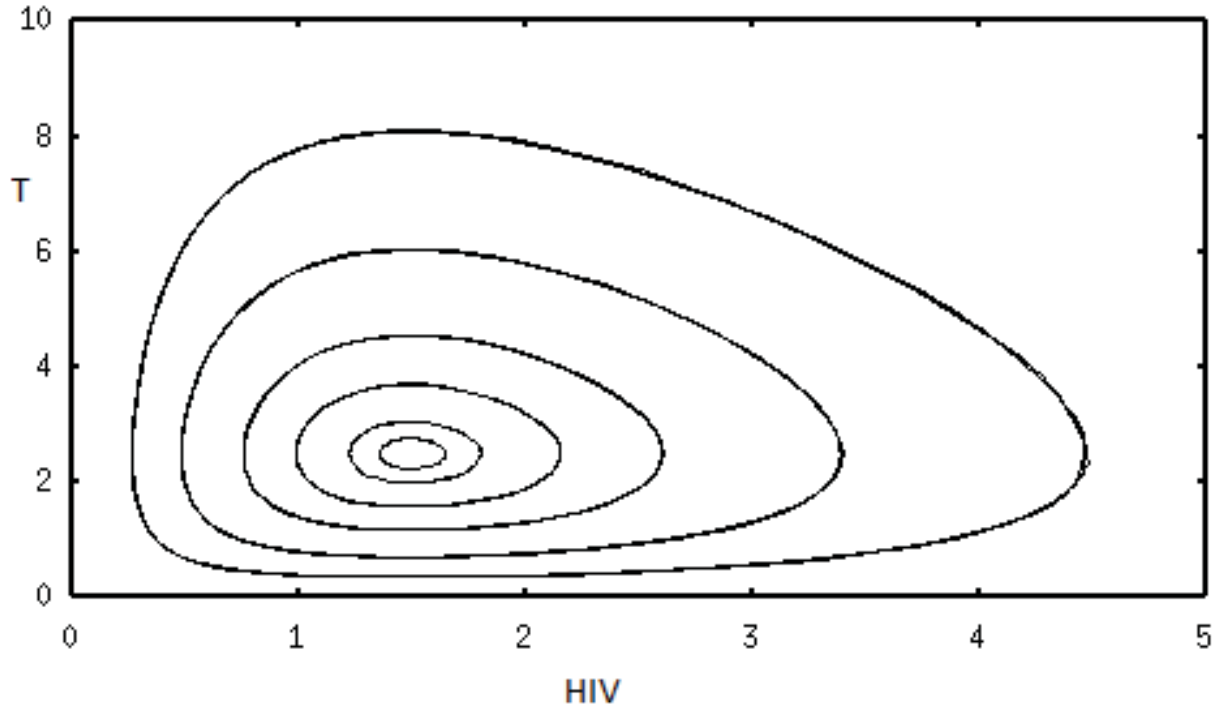

Figure 1. Phase plane topology showing a neutrally stable system for T-cells versus HIV $(\alpha=3, \beta=2, \gamma=0.01, \xi=-1, \eta=-2.5, \zeta=0.01, \varepsilon=0.01, \delta=0.01, \sigma=$ 0.01 , and $\left.\mathrm{T}_{\max }=10000.0\right)$. 


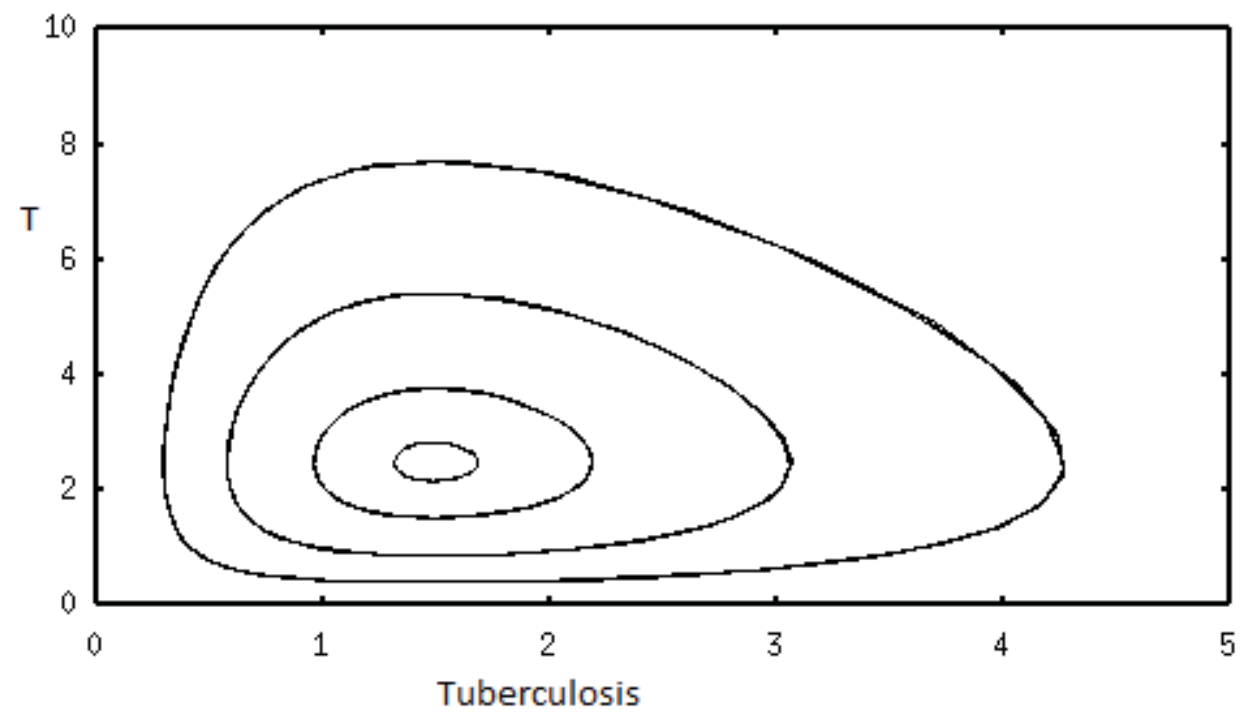

Figure 2. Phase plane topology showing a neutrally stable system for T-cells versus tuberculosis $(\alpha=3, \beta=0.01, \gamma=2.0, \xi=0.01, \eta=0.01, \zeta=0.01, \varepsilon=-1.0, \delta$ $=0.01, \sigma=-2.5$, and $\left.\mathrm{T}_{\max }=10000.0\right)$.

Figure 3 shows that the set of curves illustrating the variation of T-cells with HIV, for different initial conditions, approaching a spiral point. Similar observations are made in Figure 4 for the set of curves showing the variation of T-cells with tuberculosis. The spiral point indicates the equilibrium condition for the system. The curves approaching a spiral point represent an asymptotically stable system. ${ }^{20}$ Here, the systems for T-cells with HIV and T-cells with tuberculosis approach the equilibrium conditions at their spiral center with stability.

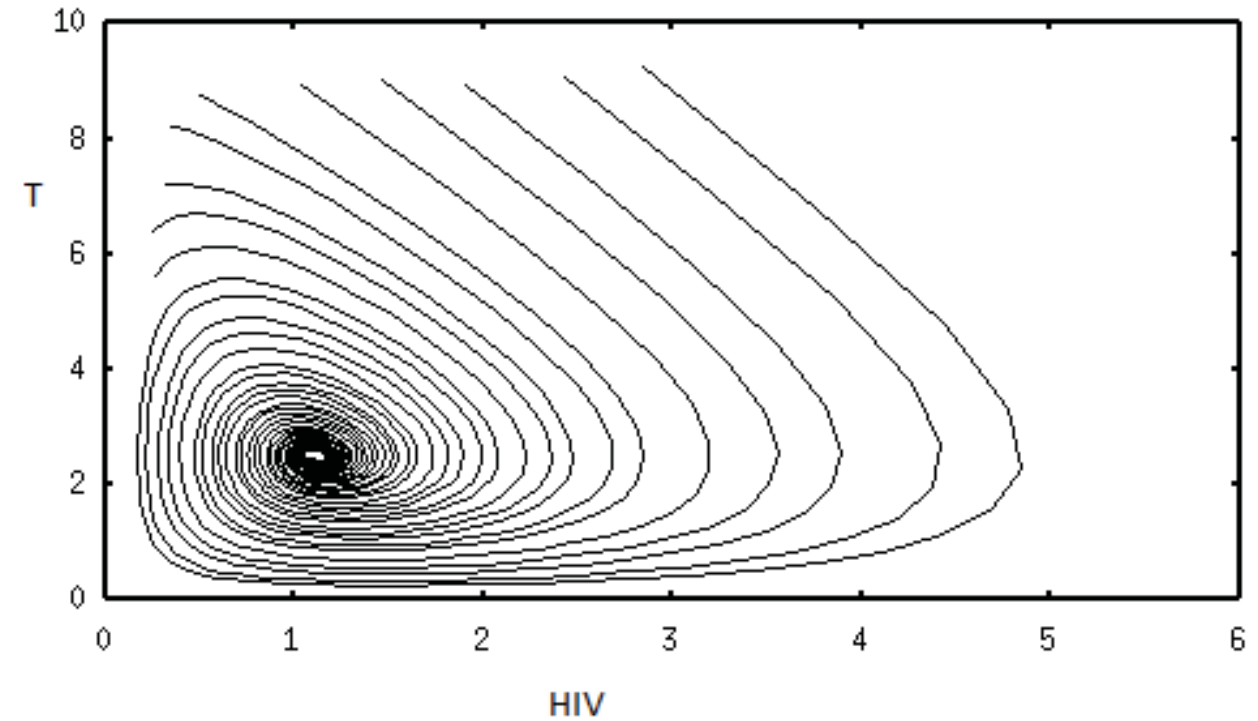

Figure 3. Phase plane topology showing an asymptotically stable system for tuberculosis versus $\operatorname{HIV}(\alpha=3, \beta=2, \gamma=0.01, \xi=-1, \eta=-2.5, \zeta=0.01, \varepsilon=0.01, \delta$ $=0.01, \sigma=0.01$, and $\left.\mathrm{T}_{\max }=10\right)$. 


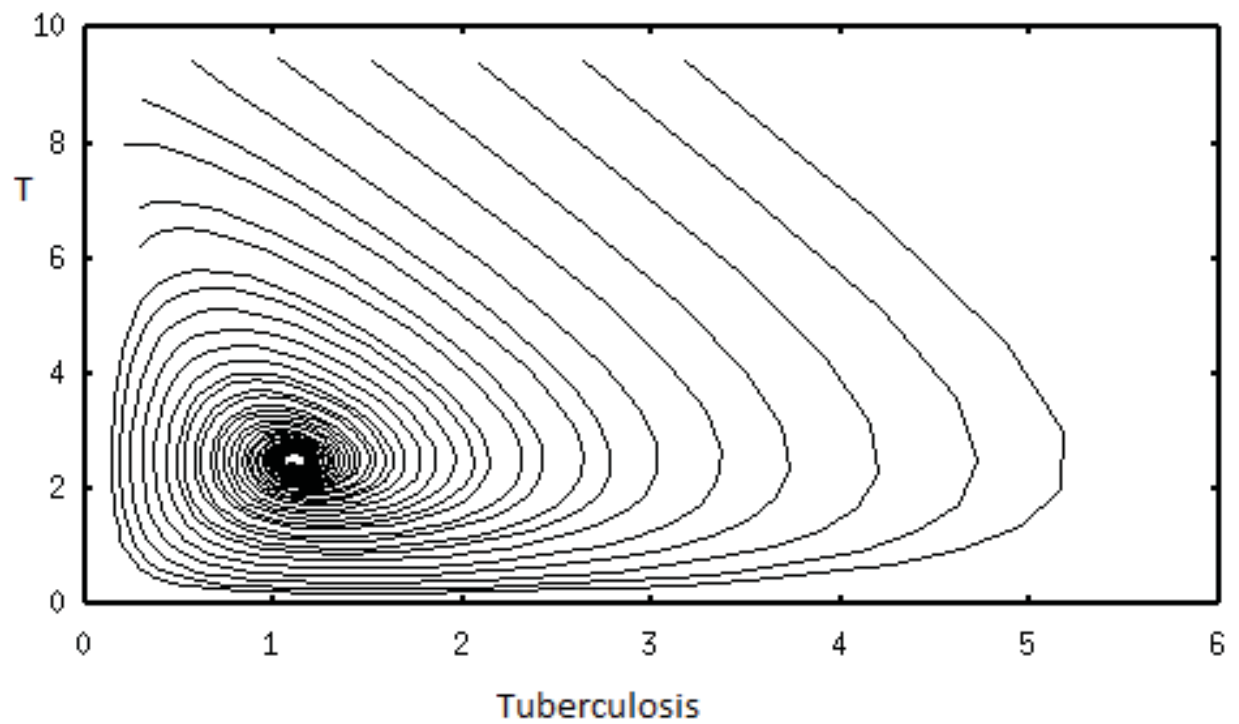

Figure 4. Phase plane topology showing an asymptotically stable system for T-cells versus tuberculosis $(\alpha=3, \beta=0.01, \gamma=2.0, \xi=0.01, \eta=0.01, \zeta=0.01, \varepsilon=$. $1.0, \delta=0.01, \sigma=-2.5$, and $\mathrm{T}_{\max }=10$ ).

Figure 5 shows the phase plane diagram illustrating the variation of T-cells with HIV. For various values of initial conditions, the set of curves shows distinct characteristics as it approaches the horizontal and vertical axes. Based on the initial conditions, the curves asymptotically approach the horizontal axis on the right side. This shows the extinction of both T-cells and HIV as $t \rightarrow \infty$. On the left side, if the initial condition for HIV is small, then the curves asymptotically approach the vertical axis with the increase of T-cells and extinction of HIV as $t \rightarrow \infty$. This is a practically possible scenario that can occur in a real life condition. Similar conditions occur in the phase diagram illustrating the variations of T-cells with tuberculosis as shown in Figure 6 . The set of parabolic curves approaching the horizontal and vertical axes in a phase plane diagram is described as an unstable system ${ }^{20}$. Here the curves shown in Figures $\mathbf{5}$ and $\mathbf{6}$, based on the set of parameter values and on the initial conditions, illustrate an unstable system.

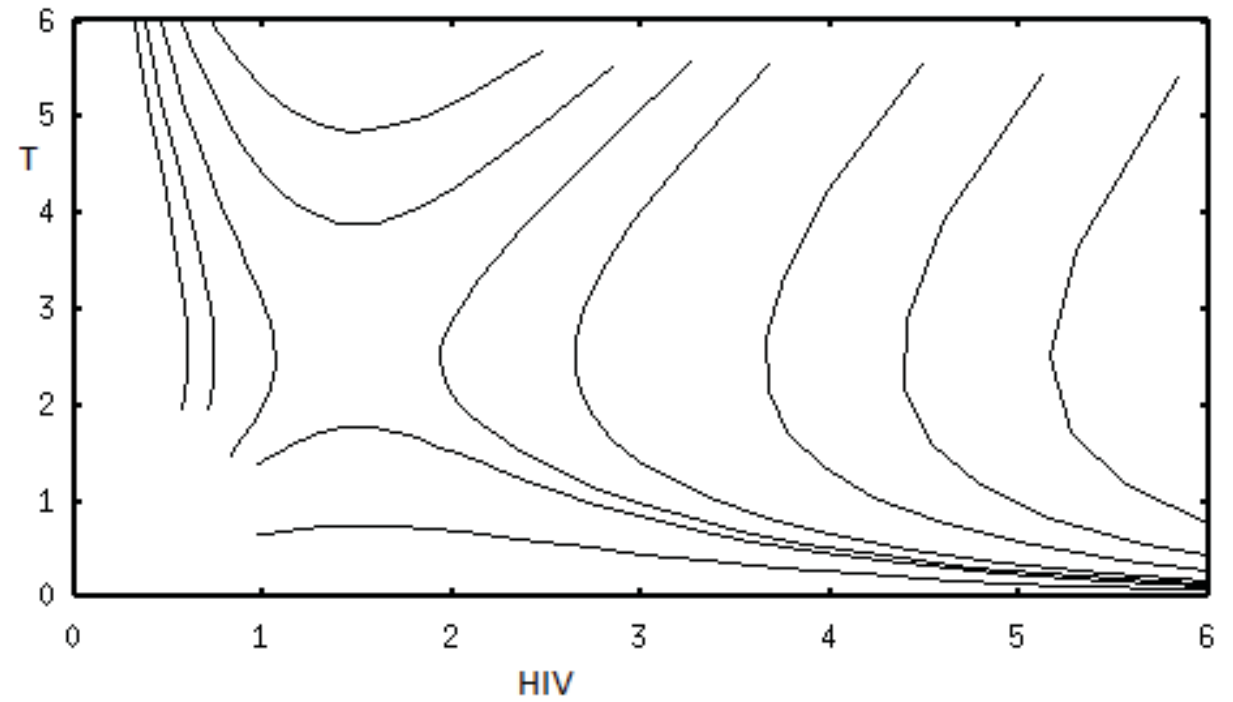

Figure 5. Phase plane topology showing an unstable system for tuberculosis versus HIV $(\alpha=3, \beta=2, \gamma=0.01, \xi=1, \eta=2.5, \zeta=0.01, \varepsilon=0.01, \delta=0.01, \sigma=$ 0.01 , and $\left.\mathrm{T}_{\max }=10000\right)$. 


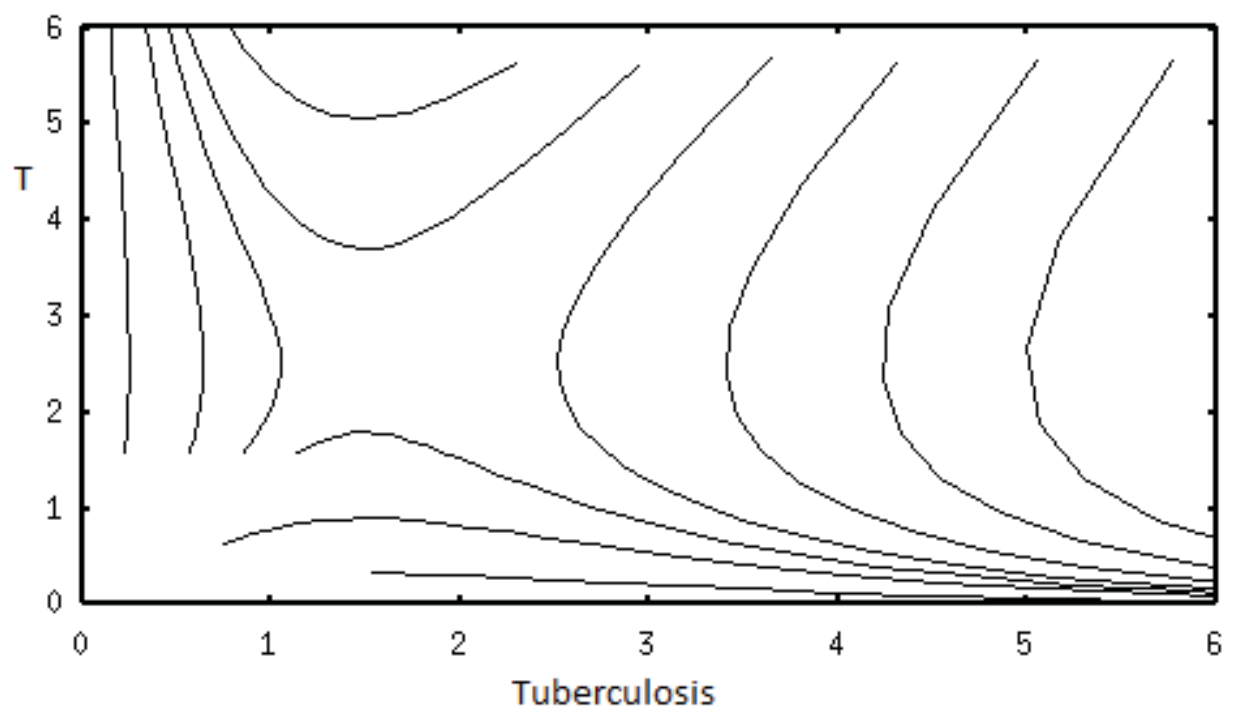

Figure 6. Phase plane topology showing an unstable system for T-cells versus tuberculosis $(\alpha=3, \beta=0.01, \gamma=2.0, \xi=0.01, \eta=0.01, \zeta=0.01, \varepsilon=1.0, \delta=$ $0.01, \sigma=2.5$, and $\left.\mathrm{T}_{\max }=10000\right)$.

\section{EXAMPLE OF UNSTEADY SIMULATIONS}

Maple software ${ }^{17}$ was used to obtain the unsteady solutions based on the Runge-Kutta method. The values of the model parameters were assigned $\left(\alpha=0.1\right.$ day $^{-1}, \beta=0.01$ day $^{-1}, \gamma=0.01$ day $^{-1}, \xi=0.01$ day $^{-1}, \eta=0.02$ day $^{-1}, \zeta=0.01$ day $^{-1}, \varepsilon=0.01$ day $^{-1}$, $\delta=0.01$ day $^{-1}$, and $\sigma=0.03$ day $^{-1}$ ) to perform the simulations for T-cells, HIV, and tuberculosis. We investigated two time varying solutions as shown in Figures 7 and 8. Different starting conditions for T-cells were considered, in order to see how they were affected by HIV and tuberculosis over time.

In Figure 7, the initial conditions are the same for all the three dependent variables (T-cells, HIV, and tuberculosis). The simulation shows how the T-cells initially increase but decline over time with the increase of HIV and tuberculosis. This shows the variation of T-cells, HIV, and tuberculosis over time. The immune cells grow but eventually vanish. At this point, HIV and tuberculosis tend to increase without bound as shown in Figure 7.

Figure 8 starts with a higher initial amount of T-cells. The adverse effects occur due to infection caused by HIV and tuberculosis. With time, the population of T-cells decline but the population of HIV and tuberculosis increase. As shown, the population of Tcells tends toward zero while the populations of HIV and tuberculosis start swiftly increasing at the end of simulation. By varying the values of the parameters, a number of solutions can be obtained to simulate the variation of T-cells, HIV, and tuberculosis with time.

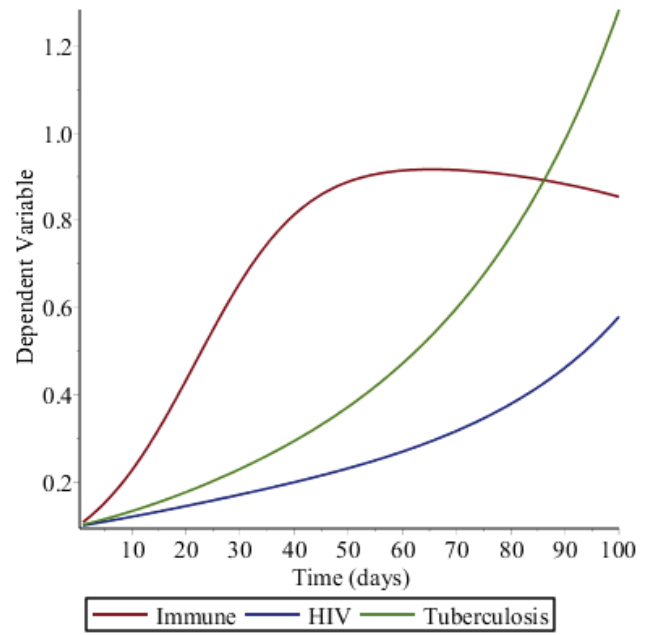

Figure 7. Simulation showing variation with time having the same starting conditions for the dependent variables. The units for T-cells (immune cells) are in thousands per cubic millimeter, the units for HIV are in thousands per milliliter, and the units for tuberculosis are in thousands CFU. 


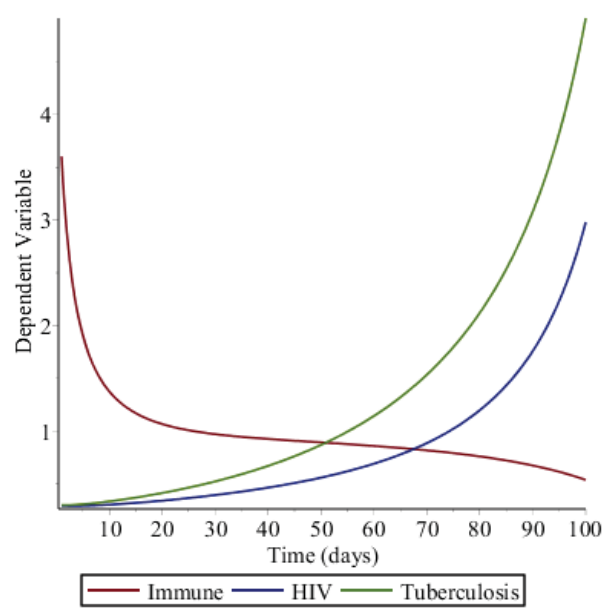

Figure 8. Simulation showing variation with time having a higher starting condition for T-cells. The units for T-cells (immune cells) are in thousands per cubic millimeter, the units for HIV are in thousands per milliliter, and the units for tuberculosis are in thousands CFU.

\section{ASSUMPTIONS, LIMITATIONS, AND FURTHER STUDY}

In the present model, it is assumed that the T-cells become infected once HIV and tuberculosis are introduced into the system. A more detailed analysis can be done by separating the T-cells into two parts; one part will represent the normal T-cells and the other part will represent the infected T-cells. This allows the simulation of the variations of normal T-cells and infected $\mathrm{T}$-cells. A separate equation can be developed and solved for the infected T-cells. The dynamical system will consist of normal T-cells, infected T-cells, HIV, and tuberculosis. Further study can be done to investigate this system with four dependent variables.

The present study entailed developing mathematical models and example applications for simulating T-cells, HIV, and tuberculosis. The mathematical models included differential equations and equilibrium solutions. The unsteady solutions are computed using a set of hypothetical values of the model parameters. We performed a few trials to determine the parameters describing the rate coefficients of the net growth rates. ${ }^{21,22}$ There are not much practical data available for the parameters involved in the system, so a practical application of the system was not performed in this study. However, a separate study can be done to examine the possibility of real world applications based on the real world data of the parameters.

\section{CONCLUSIONS}

In this study, we developed formulations for describing the unsteady variations of T-cells, HIV, and tuberculosis. To examine the stability of the system, we developed formulations based on the equilibrium solutions. We obtained and analyzed the equilibrium solutions to examine the stability of the dynamical system as time goes to infinity. The stability analysis included the investigation of the Jacobian matrix and its characteristics. Several characteristic factors involving the determinant, trace, and formulation for eigenvalues of the Jacobian matrix were developed.

Stability analysis was done by investigating the phase diagrams for T-cells varying with HIV and for T-cells varying with tuberculosis. Using sets of different parameter values, the phase planes were obtained. Graphs illustrated the growth and decay processes as time increases after the body is infected by the antigens. Graphs also illustrated the approach toward the equilibrium conditions based on different parameter values as time goes to infinity.

We investigated the stability of the dynamical system for different parameter values by examining the phase plane diagrams. We generated graphs to illustrate the stable and unstable systems as the solutions approach the equilibrium point. The phase plane diagrams showed neutrally stable systems with closed curves around a center. Along these curves, a cycle of growth and decay associated with T-cells, HIV, and tuberculosis was illustrated. Using different sets of parameter values, we obtained phase plane diagrams for unstable systems. Based on different initial conditions, these curves illustrated the progress of solutions with time.

The unsteady variations of T-cells, HIV, and tuberculosis were obtained using the assigned values of the parameters. As time increases, the growth of T-cells diminishes and gradually vanishes from the system. Through the process of the decrease of $\mathrm{T}$ cells, HIV and tuberculosis increase with time. The antigens cause unbounded vanishing of T-cells.

Further study can be performed by extending the mathematical model to four variables: normal T-cells, infected T-cells, HIV, and tuberculosis. This will require developing a differential equation for normal T-cells and infected T-cells separately. The 
equilibrium solutions, stability, and unsteady solutions can be investigated further to examine how the normal T-cells and the infected T-cells vary in a host infected by HIV and tuberculosis. Future work can include investigation of the values of the model parameters for a real world application.

\section{APPENDIX A: THE ROUTH-HURWITZ CRITERIA}

A general polynomial equation with real coefficients $a_{1}, a_{2}, a_{3}, \ldots, a_{\mathrm{n}}$ can be written as

$$
p(x)=x^{\mathrm{n}}+a_{1} x^{\mathrm{n}-1}+a_{2} x^{\mathrm{n}-2}+\cdots+a_{\mathrm{n}-1} x+a_{\mathrm{n}}=0
$$

For the polynomial $p(x), n$ Hurwitz matrices can be written as

$$
H_{1}=\left(a_{1}\right), H_{2}=\left(\begin{array}{cc}
a_{1} & 1 \\
a_{3} & a_{2}
\end{array}\right), H_{3}=\left(\begin{array}{ccc}
a_{1} & 1 & 0 \\
a_{3} & a_{2} & a_{1} \\
a_{5} & a_{4} & a_{3}
\end{array}\right), \cdots, H_{\mathrm{n}}=\left(\begin{array}{ccccc}
a_{1} & 1 & 0 & \cdots & 0 \\
a_{3} & a_{2} & a_{1} & \cdots & 0 \\
a_{5} & a_{4} & a_{3} & \cdots & 0 \\
\vdots & \vdots & \vdots & \cdots & \vdots \\
0 & 0 & 0 & \cdots & a_{\mathrm{n}}
\end{array}\right) .
$$

The roots of the polynomial equation are negative or have negative real parts if the coefficients $a_{1}, a_{2}, a_{3}, \ldots, a_{\mathrm{n}}$ and the determinants of the Hurwitz matrices are positive. The Routh-Hurwitz criteria for $\mathrm{n}=3$ can be written as $a_{i}>0, I=1,2$, and 3 .

Then $\operatorname{det} H_{2}=\operatorname{det}\left(\begin{array}{cc}a_{1} & 1 \\ a_{3} & a_{2}\end{array}\right)>0$, and so $a_{1} a_{2}>a_{3}$.

\section{REFERENCES}

1. Anderson R. M. (1994) The Croonian Lecture, 1994: Populations, Infectious Disease and Immunity: A Very Nonlinear World. Philosophical Transactions: Biological Sciences, 46:1318.

2. Doura K., Meléndez-Morales J. D., Meyer G. G., and Pérez L. E. (2000) An S-I-S Model of Streptococcal Disease with a Class of Beta-Hemolytic Carriers. Cornell University. https:/ / ecommons.cornell.edu/ bttps:// ecommons.cornell.edu/bitstream/bandle/1813/32116/BU-1524-M.pdf? sequence=1

3. Pava-Salgado E. D. L., Salguero-Rivera B., Aparicio J. P. (2001) Social Mobility and the Evolution of Tuberculosis. Cornell University. https:/ / ecommons.cornell.edu/ https:/ / ecommons.cornell.edu/bitstream/ handle/1813/32185/BU-1589-M.pdf? sequence=1

4. Pawlowski A., Jansson M., Rottenberg M. E., and Källenius G. (2012) Tuberculosis and HIV Co-Infection. Pathogens, $a$ Journal of Public Library of Science (PLOS), 8:2.

5. Pondei K. and Lawan E. (2013) Human immunodeficiency virus and pulmonary tuberculosis co-infection: Need for coordinated collaborative detection and treatment services. Journal of Medicine and Medical Sciences. 4:3, pp. 107-111.

6. Reibnegger G., Fuchs D., Hausen A., Werner E. R., Werner-Felmayer G., Dierich M. P., and Wachter H. (1989) Stability Analysis of Simple Models for Immune Cells Interacting with Normal Pathogens and Immune System Retroviruses. Proc. Natl. Acad. Sci. 86:2026-2030.

7. Akpa O. M. and Oyejola B. A. (2010) Modeling the transmission dynamics of HIV/AIDS epidemics: an introduction and a review. I Infect Dev Ctries; 4(10):597-608.

8. Alizon S. and Magnus C. (2012) Modelling the Course of an HIV Infection: Insights from Ecology and Evolution. Viruses, 4, 1984-2013.

9. Perelson A. S., and Ribeiro R. M. (2013) Modeling the within-host dynamics of HIV infection. BioMed Central (BMC), 11:96.

10. Srivastava V. K., Awasthi M. K., and Kumar S. (2014) Numerical approximation for HIV infection of CD4+ T-cells mathematical model. Engineering Physics and Mathematics, Ain Shams Engineering Journal. Vol. 5, pp. 625-629

11. Hraba T. and Dolezal J. (1996) A Mathematical Model and CD4+ Lymphocyte Dynamics in HIV Infection. Emerging Infectious Diseases, Vol. 2, No. 4, pp. 299-305.

12. Wodarz D. and Nowak M. A. (2002) Mathematical models of HIV pathogenesis and treatment. BioEssays 24:1178-1187.

13. Peñuelas-Urquides K., Villarreal-Treviño L., Silva-Ramírez B., Rivadeneyra-Espinoza L., Said-Fernández S., and León M. B. de. (2013) Measuring of Mycobacterium tuberculosis growth. A correlation of the optical measurements with colony forming units. Brazilian Journal of Microbiology. 44:1. pp. 287-289.

14. Mesterton-Gibbons M. (1995) A Concrete Approach to Mathematical Modelling. John-Wiley \& Sons. 597p. 
15. Toossi Z, Mayanja-Kizza H, Hirsch C S, Edmonds K L, Spahlinger T, Hom D L, Aung H, Mugyenyi P, Ellner J J, and Whalen C. W. (2001) Impact of Tuberculosis (TB) on HIV-1 Activity in Dually Infected Patients. Clin. Exp. Immunol. 123:2, pp. 233-238.

16. Zumla A, Malon P., Henderson J., Grange J. M. (2000) Impact of HIV Infection on Tuberculosis. Postgraduate Medical Journal. 76: pp. 259-268.

17. Maplesoft. (2014) A division of Waterloo Maple Inc. Waterloo, Canada.

18. Allen L. J. S. (2006) Introduction to Mathematical Biology. Addison-Wesley.

19. University of Pittsburg. (2016) XPPAUT: X-Windows Phase Plane Plus Auto. http:// wmw.math.pitt.edu/ $\sim$ bard/xpp/xpp.html

20. Borrelli R. L., Coleman C. S. (1998) Differential Equations - A Modeling Perspective. John Wiley \& Sons, Inc. 706p.

21. Culshaw R. V. and Ruan S. (2000) A delay-differential equation model of HIV infection of CD4+ T-cells. Mathematical Biosciences. 165. pp. 27-29.

22. Ribeiro R. M., Qin L, Chavez L. L., Li D., Self S. G., Perelson A. S. (2010) Estimation of the initial viral growth rate and basic reproductive number during acute HIV-1 infection. J. Virology. 84:12, pp. 6096-6102.

\section{ABOUT THE STUDENT AUTHOR}

Rumana Ahmed received her B.S. in Civil Engineering from the University of North Florida, Jacksonville, FL in Fall 2015. She worked as a research intern at the Mayo Clinic, Jacksonville, FL in Summer 2013. Her study included the incremental cost effectiveness ratio analysis for three methods of treatment of liver metastases. In Summer 2015, she worked as a researcher under the Travelers Summer Research Fellowship Program at Cornell University, Ithaca, New York. The research project entailed the analysis of the characteristics of carbon black-filled silicone material for pressure sensing.

For the present research paper, Rumana Ahmed worked under the guidance of Professor Mahbubur Rahman at the University of North Florida. She started working on this research in Summer 2014 and continued the work until Fall 2015. During her undergraduate years, she was actively involved as the vice president of the University of North Florida student chapter of the Society of American Military Engineers. Ms. Ahmed worked as a project engineer with Fusion Engineering, New York in the areas of hydraulic and environmental engineering. Currently she is a graduate student pursuing her M.S. in biology at the City College of the City University of New York since Fall 2016.

\section{PRESS SUMMARY}

Mathematical biology is an evolving area where mathematical and numerical analyses are performed to simulate the biological processes. It is a challenge to develop stable formulations for simulating the growth-decay and the interaction processes in a biological system. In this study, formulations based on ordinary differential equations have been developed by incorporating growth-decay and interaction of the population of blood cells and antigens in a host. This research focused on the simulation of the dynamics of immune cells, HIV, and tuberculosis. Equilibrium solutions were developed and investigated as time goes to infinity. The criteria for the stability of the dynamical system have been established. In addition, stability analysis has been performed by investigating the phase plane diagrams. Finally, the unsteady state solutions were generated to simulate the variations of T-cells, HIV, and tuberculosis with time. 